\title{
Synthesis of 1,3-cis-disubstituted sterically encumbered imidazolidinone organocatalysts
}

\author{
Jan Wallbaum and Daniel B. Werz
}

\author{
Full Research Paper \\ Address: \\ Institut für Organische Chemie, Technische Universität Braunschweig, \\ Hagenring 30, 38106 Braunschweig, Germany \\ Email: \\ Daniel B.Werz - d.werz@tu-braunschweig.de \\ * Corresponding author \\ Keywords: \\ imidazolidinone; MacMillan catalyst; organocatalysis
}

\author{
Beilstein J. Org. Chem. 2017, 13, 2577-2583. \\ doi:10.3762/bjoc. 13.254 \\ Received: 25 October 2017 \\ Accepted: 20 November 2017 \\ Published: 01 December 2017 \\ Associate Editor: J. S. Dickschat \\ (C) 2017 Wallbaum and Werz; licensee Beilstein-Institut. \\ License and terms: see end of document.
}

\begin{abstract}
A variety of novel imidazolidinone-based organocatalysts with bulky substituents were synthesized under mild reaction conditions starting from easily accessible substrates. Different natural and unnatural amino acid methyl amides were cyclized with aromatic carbaldehydes to yield two diastereomeric MacMillan-type catalysts. Special emphasis was put on bulky residues such as mesityl and pyrene moieties.
\end{abstract}

\section{Introduction}

Organocatalytic iminium and enamine activation has attracted organic chemists for more than one century [1]. Until today a wide and constantly increasing number of different organocatalytic transformations with various substrates have been accomplished [2-7]. Initially, proline and proline-derived catalysts have been widely used in asymmetric iminium and enamine organocatalysis [8-12]. Since the beginning of the 21 th century imidazolidinone-based organocatalysts developed by MacMillan and co-workers, which are easily accessible from amino acids, are widely used in these kinds of reactions [13-18]. More recent examples demonstrate the applicability in various reactions like diastereoselective $\alpha$-fluorination [19], total syntheses [20,21], cross-dehydrogenative couplings [22], selec- tivity-reversed Friedel-Crafts alkylation [23] and in combination with photoredox catalysis (Scheme 1a) [24].

The enantioselective $\alpha$-alkylation was achieved by merging the common photoredox catalyst $\mathrm{Ru}(\mathrm{bpy}){ }_{3} \mathrm{Cl}_{2}$ with imidazolidinone catalyst $3 \mathbf{a} \cdot \mathrm{TfOH}$, controlling the stereochemistry of the radical addition via an intermediate enamine complex.

Scheme $1 \mathrm{~b}$ shows our regio-, diastereo- and enantioselective 1,3-chlorosulfenation of meso-cyclopropyl carbaldehydes employing a newly designed organocatalyst $7 \mathbf{a} \cdot \mathrm{DCA}$ for chiral induction [25]. In the course of these studies we prepared a variety of imidazolidinone organocatalysts with rather bulky 
a)

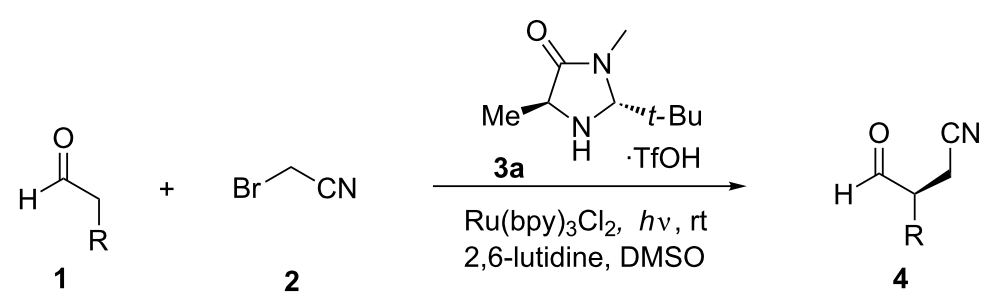

b)<smiles>[R]C1C([R])C1[18O]</smiles>

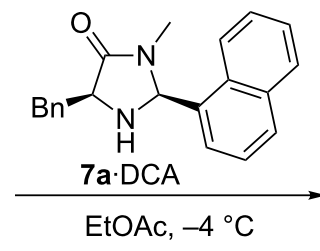<smiles>[R]C(Cl)C([R1])C([R])[Hg]</smiles>

(er up to 9:1)

Scheme 1: a) MacMillan's enantioselective a-alkylation of aldehydes. b) Our enantioselective 1,3-chlorosulfenation of meso-cyclopropyl carbaldehydes.

substituents. In this paper we report on these MacMillan-type catalysts which will be of great value for the screening of further transformations based on iminium-enamine mechanisms.

\section{Results and Discussion}

In order to screen a certain transformation by using a variety of organocatalysts with subtle differences in the substitution pattern a modular approach for their preparation is highly desirable. A simple retrosynthetic cut of the five-membered ring delivers an amino acid methyl amide and a carbaldehyde as starting materials. Chirality is commonly introduced by the use of derivatives of naturally occurring L-amino acid derivatives. During the condensation process two diastereomers can be formed with the substituents in either a 1,3-cis or 1,3-trans arrangement. Since our reaction design of the above-mentioned reaction (depicted in Scheme 1b) showed a strong preference to use the 1,3-cis-disubstituted derivatives we concentrated our efforts on the isolation of these isomers.

Methyl amides 9 were produced from the corresponding methyl or ethyl esters via reaction with ethanolic methyl amide solution [26]. Solid methyl amides were further recrystallized to achieve a higher purity. Protected methyl amides 9a and 9c were synthesized in five or three literature-known steps from commercially available substrates, respectively [27-29].

The different methyl amides were subjected to the reaction with 1-naphthyl carbaldehyde employing $4 \AA$ molecular sieves as dehydrating agent and $10 \mathrm{~mol} \% \mathrm{Yb}(\mathrm{OTf})_{3}$ in THF. This Lewis acid proved to be the Lewis acid of choice since the reaction proceeds without loss of optical purity [30]. The naphthyl residue was chosen since it revealed a high selectivity in the desired organocatalytic transformation. Table 1 depicts the scope with respect to 1-naphthyl carbaldehyde. Crude ${ }^{1} \mathrm{H}$ NMR data of the reaction mixture showed in every case approximately $90 \%$ product formation of both diastereomers together, using an internal standard. The corresponding 1,3-trans diastereomers were not isolated in pure form. As reaction partners different methyl amides 9a-f were employed in the reaction. The use of methyl-protected L-histidine-derived methyl amide 9a (Table 1, entry 1) gave the desired imidazolidinone $\mathbf{7 b}$ in $42 \%$ yield, whereas the unprotected derivative showed no conversion, probably because of its insolubility. The unprotected as well as the benzyl-protected L-tryptophan-derived methyl amides $9 \mathbf{b}$ and $9 \mathbf{c}$ were converted in good yields to the corresponding imidazolidinones (Table 1, entries 2 and 3). In the case of $7 \mathbf{c}$ it is important to use not more than 1.0 equivalent of the aldehyde, otherwise a further reaction with the indole moiety is observed.

A significant change in selectivity was observed while using electron-withdrawing methyl amide 9d; the influence of the para-nitro substituent decreases the yield of the desired cis-diastereomer to only $12 \%$. Utilization of non-aromatic methyl amides 9e and 9f, synthesized from L-norvaline and L-methionine methyl esters, gave rise to the desired imidazolidinones $7 \mathrm{f}$ and $7 \mathrm{~g}$ (Table 1, entries 5 and 6) in $46 \%$ and $32 \%$, respectively.

After evaluating the scope of imidazolidinones 7 we were keen to investigate which sterically more demanding aldehydes 
Table 1: Scope of the reaction using 1-naphthyl carbaldehyde (10a). ${ }^{a}$<smiles></smiles>

$\mathrm{Yb}(\mathrm{OTf})_{3}(10 \mathrm{~mol} \%)$

$4 \AA \mathrm{MS}, \mathrm{THF}, \mathrm{rt}$

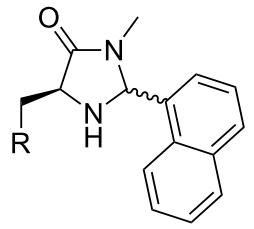

7

\begin{tabular}{llll}
\hline Entry & Methyl amide 9 & Imidazolidinone 7 & Yield [\%] (ratio) \\
\hline
\end{tabular}

1<smiles>CNC(=O)[C@H](N)Cc1cn(C)cn1</smiles>

2<smiles>CNC(=O)[C@H](N)Cc1c[nH]c2ccccc12</smiles>

3<smiles>CNC(=O)[C@H](N)Cc1c[nH]c2ccccc12</smiles>

4<smiles>CNC(=O)[C@H](N)Cc1ccc([N+](=O)[O-])cc1</smiles>

5<smiles>CCC[C@H](N)C(=O)NC</smiles>

6
$9 a$<smiles>CN1C(=O)[C@@H](Cc2cn(C)cn2)N[C@H]1c1cccc2ccccc12</smiles>

$7 b$<smiles>CN1C(=O)[C@@H](Cc2c[nH]c3ccccc23)N[C@H]1c1cccc2ccccc12</smiles>

9c<smiles>CN1C(=O)[C@@H](Cc2c[nH]c3ccccc23)N[C@@H]1c1cccc2ccccc12</smiles>

9d<smiles>CN1C(=O)[C@@H](Cc2ccc([N+](=O)[O-])cc2)N[C@H]1c1cccc2ccccc12</smiles>

$9 e$<smiles>CN1C(=O)[C@H](P=P)N[C@H]1c1cccc2ccccc12</smiles>

$9 f$
$7 e$

$7 g$<smiles>CSCC[C@H]1N[C@@H](c2cccc3ccccc23)N(C)C1=O</smiles>

aReaction conditions: 9 (1.0 equiv), 10a (0.9-1.1 equiv), Yb(OTf) 3 (10 mol \%), THF (4-12 mL/mmol), $4 \AA \mathrm{MS}(40 \mathrm{mg} / \mathrm{mL}), \mathrm{rt}, 24 \mathrm{~h} .{ }^{\mathrm{b}} / \mathrm{solated}$ yield after column chromatography; the yield of both diastereomers together is in every case about $80-90 \%$; the ratio of the two diastereomers is given in brackets, the desired cis-isomer is underlined (determined via ${ }^{1} \mathrm{H}$ NMR spectroscopy of the crude reaction mixture).

could be employed. Since the anticipated organocatalytic transformation (shown in Scheme 1b) delivered relatively high selectivity with imidazolidinones derived from L-phenylalanine this amino acid derivative was used as coupling partner. 1-Pyrene carbaldehyde 10b gave only a minor excess of the cis-diastereomer $(S, S)$-3b. For mesityl carbaldehyde 10c the desired isomer was obtained in $52 \%$ yield whereas the more sterically demanding aldehyde 10d led to a switch in the selectivity, giving the desired $(S, S)$-diastereomer in only $23 \%$ yield (Table 2).

\section{Conclusion}

We were able to synthesize 10 different imidazolidone-based organocatalysts with yields of the desired 1,3-cis-disubstituted diastereomers of up to $52 \%$. Different methyl amides derived from $\alpha$-amino acids and bulky aromatic carbaldehydes were employed to access the MacMillan-type catalysts. The key to the success is the activation with ytterbium triflate as Lewis acid and the use of dehydrating molecular sieves. The prepared organocatalysts will be a useful contribution for the screening of a multitude of different organocatalytic transformations. 
Table 2: Scope of the imidazolidinone formation with respect to aldehyde $10{ }^{\text {a }}$

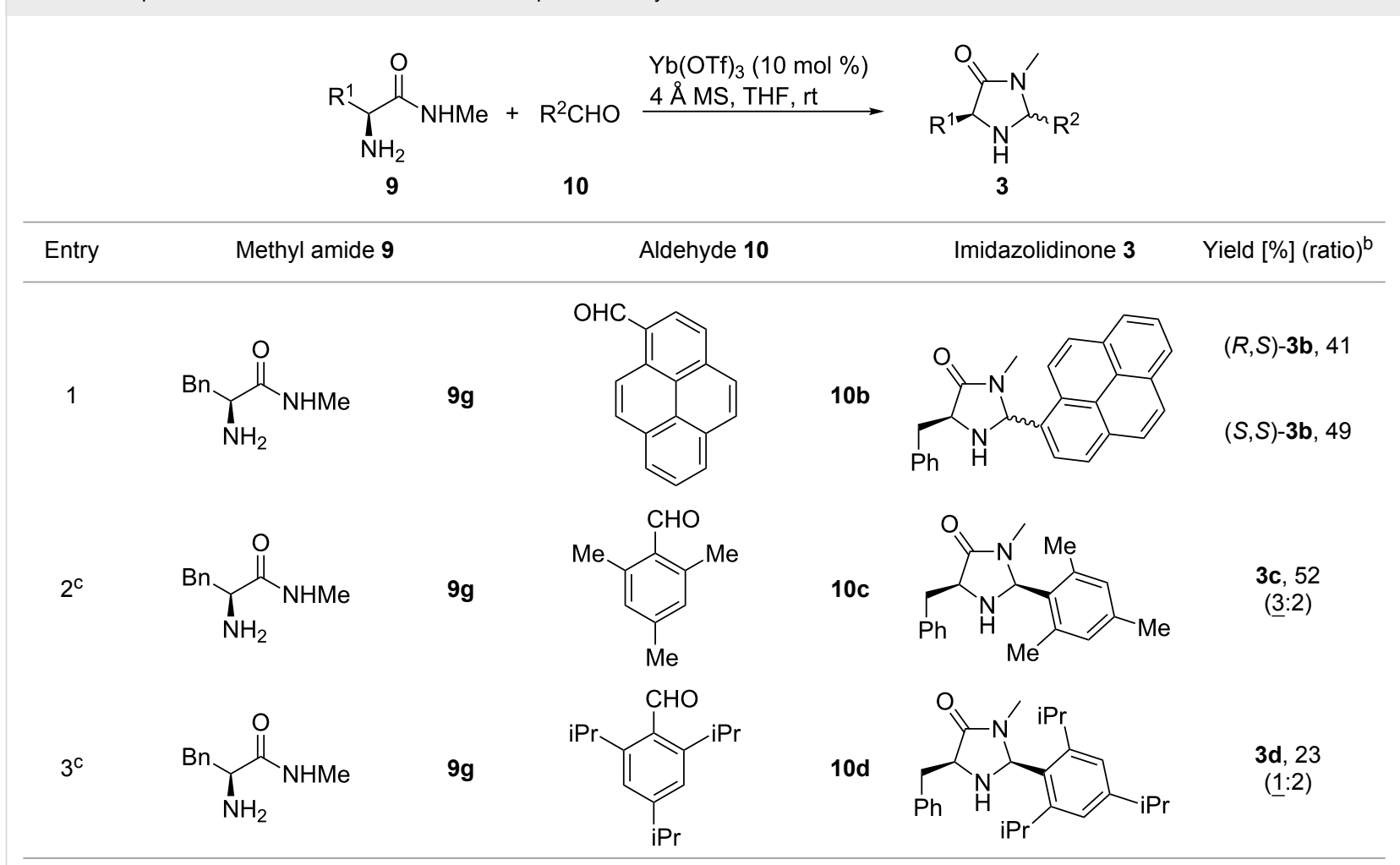

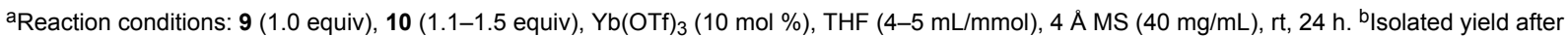
column chromatography. The ratio of the two diastereomers is given in brackets, the desired cis-isomer is underlined (determined via ${ }^{1} \mathrm{H}$ NMR spectroscopy of the crude reaction mixture). ${ }^{c}$ Combined yield of both diastereomers is $89 \%$ (entry 2 ) and $78 \%$ (entry 3 ), respectively.

\section{Experimental}

General. All solvents were distilled before use unless otherwise stated. Tetrahydrofuran (THF) was distilled over sodium and benzophenone under an argon atmosphere. Air- and moisture-sensitive reactions were carried out in oven-dried or flamedried glassware, septum-capped under atmospheric pressure of argon. Commercially available compounds were used without further purification unless otherwise stated.

Proton $\left({ }^{1} \mathrm{H}\right)$ and carbon $\left({ }^{13} \mathrm{C}\right)$ NMR spectra were recorded on a 300,400 or $600 \mathrm{MHz}$ instrument using the residual signals from $\mathrm{CHCl}_{3}, \delta=7.26 \mathrm{ppm}$ and $\delta=77.0 \mathrm{ppm}$, or $\mathrm{MeOH}$, $\delta=3.31 \mathrm{ppm}$ and $\delta=49.2 \mathrm{ppm}$, using TMS as internal reference for ${ }^{1} \mathrm{H}$ and ${ }^{13} \mathrm{C}$ chemical shifts, respectively. Assignments of the respective signals and the stereochemistry were made by combination of H,H-COSY, HSQC, HMBC and NOESY experiments. In some cases signals in the ${ }^{13} \mathrm{C}$ NMR spectrum are missing because of bad relaxation of the respective signals. ESI high-resolution mass spectrometry was carried out on a FTICR instrument. IR spectra were measured on an ATR spectrometer. Optical rotation was measured on a common polarimeter.
General procedure (GP) for the synthesis of imidazolidinones of type 3 and 7 . A Schlenk flask was charged with a magnetic stirring bar and powdered $4 \AA$ molecular sieves $(20 \mathrm{mg} / \mathrm{mL})$ and flame-dried for 10 minutes under high vacuum. After cooling to ambient temperature methyl amide (1.0 equiv) was added and the flask subjected to the glovebox. After addition of $\mathrm{Yb}(\mathrm{OTf})_{3}(10 \mathrm{~mol} \%)$ the flask was removed from the glovebox and THF (4.0 to $12.0 \mathrm{~mL} / \mathrm{mmol}$ ) and the corresponding aldehyde ( 0.9 to 1.2 equiv) were added subsequently. The resulting mixture was stirred at ambient temperature for $24 \mathrm{~h}$. The suspension was filtered over Celite and the solvent was removed in vacuo.

(2S,5S)-5-((N-Methyl-1H-imidazol-4-yl)methyl)-3-methyl-2(naphthalen-1-yl)imidazolidin-4-one (7b). ( $S$ )- $N$-Methylhistidine methyl amide (190 mg, $1.04 \mathrm{mmol}, 1.0$ equiv), 1-naphthyl carbaldehyde (180 mg, $156 \mu \mathrm{L}, 1.15 \mathrm{mmol}, 1.1$ equiv), $4 \AA$ molecular sieves $(240 \mathrm{mg})$ and $\mathrm{Yb}(\mathrm{OTf})_{3}(64.5 \mathrm{mg}, 104 \mu \mathrm{mol}$, $10 \mathrm{~mol} \%)$ in THF (12 mL) were reacted according to the GP. Silica gel column chromatography (DCM/MeOH 1:0 $\rightarrow$ 50:1) afforded the desired $(S, S)$-diastereomer $7 \mathbf{b}(140 \mathrm{mg}, 437 \mu \mathrm{mol}$, $42 \%$ ) as pale yellow oil: ${ }^{1} \mathrm{H}$ NMR (200 MHz, MeOD) $\delta 2.65$ (s, 
$3 \mathrm{H}), 3.05(\mathrm{dd}, J=9.4,5.1 \mathrm{~Hz}, 2 \mathrm{H}), 3.70(\mathrm{~s}, 3 \mathrm{H}), 3.91(\mathrm{t}, J=$ $5.1 \mathrm{~Hz}, 1 \mathrm{H}), 6.09$ (br s, 1H), 6.87-6.98 (m, 1H), 6.97-7.12 (m, $1 \mathrm{H}), 7.43-7.58(\mathrm{~m}, 4 \mathrm{H}), 7.84-8.00(\mathrm{~m}, 2 \mathrm{H}), 8.02-8.16(\mathrm{~m}, 1 \mathrm{H})$; ${ }^{13} \mathrm{C}$ NMR $\left(150 \mathrm{MHz}, \mathrm{CDCl}_{3}\right) \delta 28.7,33.3,60.4,60.4,118.3$, $122.7,125.1,125.1,126.0,126.6,126.7,128.8,137.2,137.3$, 137.8 (the carbonyl signal as well as one aliphatic, one aromatic $\mathrm{CH}$ and one aromatic $\mathrm{C}_{\mathrm{q}}$-signal are missing); $\alpha_{\mathrm{D}}^{24.0}$ (c 1.0, $\left.\mathrm{CHCl}_{3}\right)+38.0^{\circ}$; IR (ATR) $\tilde{v}\left(\mathrm{~cm}^{-1}\right): 3349,2922,1683,1261$, 1159; HRMS (ESI) $m / z$ : calcd for $\mathrm{C}_{19} \mathrm{H}_{20} \mathrm{~N}_{4} \mathrm{O}, 343.1529$; found, 343.1529 .

(2S,5S)-5-((Indol-3-yl)methyl)-3-methyl-2-(naphthalen-1yl)imidazolidin-4-one (7c). ( $S$ )-Tryptophan methyl amide (340 mg, $1.56 \mathrm{mmol}, 1.0$ equiv), 1-naphthyl carbaldehyde (243 mg, $212 \mu \mathrm{L}, 1.56 \mathrm{mmol}, 1.0$ equiv), $4 \AA$ molecular sieves $(160 \mathrm{mg})$ and $\mathrm{Yb}(\mathrm{OTf})_{3}(97.0 \mathrm{mg}, 156 \mu \mathrm{mol}, 10 \mathrm{~mol} \%)$ in THF $(8.0 \mathrm{~mL})$ were reacted according to the GP. Silica gel column chromatography ( $n$-pentane/EtOAc 1:1 $\rightarrow 2: 3 \rightarrow 0: 1$ ) gave the desired $(S, S)$-diastereomer 7c $(250 \mathrm{mg}, 203 \mu \mathrm{mol}, 45 \%)$ as pale yellow solid: $\mathrm{mp} 145{ }^{\circ} \mathrm{C} ;{ }^{1} \mathrm{H}$ NMR (300 MHz, MeOD) $\delta 2.52$ (s, 3H), 3.23 (dd, $J=14.8,5.3 \mathrm{~Hz}, 1 \mathrm{H}), 3.49$ (dd, $J=14.8,3.9$ $\mathrm{Hz}, 1 \mathrm{H}), 3.85-3.96(\mathrm{~m}, 1 \mathrm{H}), 5.98$ (br s, 1H), 6.86-6.98 (m, 1H), 6.99-7.17 (m, 4H), 7.33-7.47 (m, 3H), $7.53(\mathrm{~d}, J=7.9 \mathrm{~Hz}, 1 \mathrm{H})$, 7.69-7.87 (m, 3H); ${ }^{13} \mathrm{C}$ NMR (75 MHz, MeOD) $\delta 30.6,30.6$, $64.3,112.2,115.0,122.3,122.7,125.3,125.6,128.2,128.9$, 129.6, 130.3, 131.6, 132.5, 133.3, 133.4, 134.8, 137.9, 140.9, 180.3 (one aliphatic $\mathrm{CH}$ and one aromatic $\mathrm{CH}$ are missing); $\alpha_{\mathrm{D}}^{24.0}\left(c\right.$ 1.0, $\left.\mathrm{CHCl}_{3}\right)-6.0^{\circ}$; IR (ATR) $\tilde{v}\left(\mathrm{~cm}^{-1}\right): 3404,3280$, 1681, 1435, 1097; HRMS (ESI) $m / z$ : calcd for $\mathrm{C}_{23} \mathrm{H}_{21} \mathrm{~N}_{3} \mathrm{O}$, 378.1577 ; found, 378.1578 .

(2S,5S)-5-((N-Benzylindol-3-yl)methyl)-3-methyl-2-(naphthalen1-yl)imidazolidin-4-one (7d). $(S)$ - $N$-Benzyltryptophan methyl amide (307 mg, $1.00 \mathrm{mmol}, 1.0$ equiv), 1-naphthyl carbaldehyde (156 mg, $136 \mu \mathrm{L}, 1.10 \mathrm{mmol}, 1.1$ equiv), $4 \AA$ molecular sieves $(120 \mathrm{mg})$ and $\mathrm{Yb}(\mathrm{OTf})_{3}(62.0 \mathrm{mg}, 100 \mu \mathrm{mol}$, $10 \mathrm{~mol} \%)$ in THF $(6.0 \mathrm{~mL})$ were reacted according to the GP. Silica gel column chromatography $(n$-pentane/EtOAc 1:0 $\rightarrow$ 50:1) the desired $(S, S)$-diastereomer 7d (188 mg, $422 \mu \mathrm{mol}$, $42 \%$ ) as pale yellow solid: $\mathrm{mp} 120{ }^{\circ} \mathrm{C}(\mathrm{dec}) ;{ }^{1} \mathrm{H}$ NMR (200 MHz, MeOD) $\delta 2.54(\mathrm{~s}, 3 \mathrm{H}), 3.11-3.32(\mathrm{dd}, J=14.9$, $5.5 \mathrm{~Hz}, 1 \mathrm{H}), 3.51(\mathrm{dd}, J=14.9,3.6 \mathrm{~Hz}, 1 \mathrm{H}), 3.88-3.96(\mathrm{~m}, 1 \mathrm{H})$, $5.23(\mathrm{~s}, 2 \mathrm{H}), 6.01$ (br s, 1H), 6.87-7.22 (m, 11H), 7.32-7.48 (m, $3 \mathrm{H}), 7.55(\mathrm{~d}, J=7.9 \mathrm{~Hz}, 1 \mathrm{H}), 7.71(\mathrm{~d}, J=8.3 \mathrm{~Hz}, 1 \mathrm{H}), 7.83$ (d, $J=8.4 \mathrm{~Hz}, 1 \mathrm{H}) ;{ }^{13} \mathrm{C} \mathrm{NMR}\left(150 \mathrm{MHz}, \mathrm{CDCl}_{3}\right) \delta 26.3,27.9$, 49.9, 60.3, 109.3, 109.6, 119.6, 122.0, 122.3, 125.3, 126.0, $126.7,126.7,127.5,127.8,128.3,128.6,128.8,131.0,133.7$, 136.8, 137.2, 176.1 (one aliphatic, two aromatic $\mathrm{CH}$ and one aromatic $\mathrm{C}_{\mathrm{q}}$-signals are missing); $\alpha_{\mathrm{D}}^{24.0}\left(c 1.0, \mathrm{CHCl}_{3}\right)-6.0^{\circ}$; IR (ATR) $\tilde{v}\left(\mathrm{~cm}^{-1}\right): 3491,1684,1259,1170,1035$; HRMS (ESI) $\mathrm{m} / \mathrm{z}$ : calcd for $\mathrm{C}_{30} \mathrm{H}_{27} \mathrm{~N}_{3} \mathrm{O}$, 468.2046; found, 468.2050.
(2S,5S)-5-(p-Nitrobenzyl)-3-methyl-2-(naphthalen-1yl)imidazolidin-4-one (7e). ( $S$ )-p-Nitrophenylalanine methyl amide (892 mg, $4.00 \mathrm{mmol}, 1.0$ equiv), 1-naphthyl carbaldehyde (625 mg, $543 \mu \mathrm{L}, 4.00 \mathrm{mmol}, 1.0$ equiv), $4 \AA$ molecular sieves $(480 \mathrm{mg})$ and $\mathrm{Yb}(\mathrm{OTf})_{3}(248 \mathrm{mg}, 400 \mu \mathrm{mol}, 10 \mathrm{~mol} \%)$ in THF $(24 \mathrm{~mL})$ were reacted according to the GP. Silica gel column chromatography ( $n$-pentane/EtOAc $2: 1 \rightarrow 1: 1 \rightarrow 0: 1$ ) gave the desired $(S, S)$-diastereomer $7 \mathrm{e}(180 \mathrm{mg}, 498 \mu \mathrm{mol}$, $12 \%)$ as pale yellow solid: mp $75{ }^{\circ} \mathrm{C} ;{ }^{1} \mathrm{H}$ NMR $(300 \mathrm{MHz}$, $\left.\mathrm{CDCl}_{3}\right) \delta 2.71(\mathrm{~s}, 3 \mathrm{H}), 3.16-3.37(\mathrm{~m}, 2 \mathrm{H}), 4.02(\mathrm{dd}, J=5.1$, $5.1 \mathrm{~Hz}, 1 \mathrm{H}), 5.99$ (br s, 1H), 7.02 (br s, 1H), 7.28-7.41 (m, 3H), 7.41- $7.57(\mathrm{~m}, 2 \mathrm{H}), 7.79-7.94(\mathrm{~m}, 2 \mathrm{H}), 7.94-8.06(\mathrm{~m}, 1 \mathrm{H})$, 8.05-8.13 (m, 2H); $\left.{ }^{13} \mathrm{C} \mathrm{NMR} \mathrm{(75} \mathrm{MHz,} \mathrm{CDCl}_{3}\right) \delta 28.0,37.3$, $60.4,77.2,122.3,123.7,125.2,126.2,126.8,129.1,130.0$, $130.6,131.0,134.0,144.9,147.0$ (the carbonyl and two aromatic $\mathrm{CH}$ signals are missing); $\alpha_{\mathrm{D}}^{24.0}\left(c 1.0, \mathrm{CHCl}_{3}\right)-26.0^{\circ}$; IR (ATR) $\tilde{v}\left(\mathrm{~cm}^{-1}\right): 3324,1690,1513,1342,1107$; HRMS (ESI) $\mathrm{m} / \mathrm{z}$ : calcd for $\mathrm{C}_{21} \mathrm{H}_{19} \mathrm{~N}_{3} \mathrm{O}_{3}, 384.1319$; found, 384.1319 .

(2S,5S)-3-Methyl-2-(naphthalen-1-yl)-5-propylimidazolidin4-one (7f). (S)-Norvaline methyl amide $(521 \mathrm{mg}, 4.00 \mu \mathrm{mol}$, 1.0 equiv), 1-naphthyl carbaldehyde (562 mg, $489 \mu \mathrm{L}$, $3.60 \mathrm{mmol}, 0.9$ equiv), $4 \AA$ molecular sieves $(480 \mathrm{mg})$ and $\mathrm{Yb}(\mathrm{OTf})_{3}(248 \mathrm{mg}, 400 \mu \mathrm{mol}, 10 \mathrm{~mol} \%)$ in THF $(24 \mathrm{~mL})$ were reacted according to GP. Silica gel column chromatography ( $n$-pentane/EtOAc 4:1 $\rightarrow$ 1:1) gave the desired $(S, S)$-diastereomer $7 f$ (440 mg, $1.64 \mathrm{mmol}, 46 \%)$ as pale yellow oil. ${ }^{1} \mathrm{H}$ NMR $\left(200 \mathrm{MHz}, \mathrm{CDCl}_{3}\right) \delta 0.94(\mathrm{t}, J=7.2 \mathrm{~Hz}, 3 \mathrm{H}), 1.34-1.71(\mathrm{~m}$, $3 \mathrm{H}), 1.87$ (br s, 1H, NH), 1.89-2.13 (m, 1H), 3.57-3.70 (m, $1 \mathrm{H}), 6.01$ (br s, 1H), 7.40-7.61 (m, 4H), 7.83-8.00 (m, 2H), 8.15-8.29 (m, 1H); $\left.{ }^{13} \mathrm{C} \mathrm{NMR} \mathrm{(75} \mathrm{MHz}, \mathrm{CDCl}_{3}\right) \delta 13.9,19.2$, 28.1, 34.4, 59.8, 77.2, 122.6, 125.4, 126.2, 126.9, 129.0, 129.8, $131.2,131.4,133.7,134.1,176.5 ; \alpha_{\mathrm{D}}^{24.0}\left(c\right.$ 1.0, $\left.\mathrm{CHCl}_{3}\right)+89.0^{\circ}$; IR (ATR) $\tilde{v}\left(\mathrm{~cm}^{-1}\right): 3321,2957,1689,1396,1322$; HRMS (ESI) $m / z$ : calcd for $\mathrm{C}_{17} \mathrm{H}_{20} \mathrm{~N}_{2} \mathrm{O}, 291.1468$; found, 291.1468.

(2S,5S)-3-Methyl-5-(2-(methylthio)ethyl)-2-(naphthalen-1yl)imidazolidin-4-one (7g). ( $S$ )-Methionine methyl amide (649 mg, $4.00 \mu \mathrm{mol}, 1.0$ equiv), 1-naphthyl carbaldehyde (562 mg, $489 \mu \mathrm{L}, 3.60 \mathrm{mmol}, 0.9$ equiv), $4 \AA$ molecular sieves $(480 \mathrm{mg})$ and $\mathrm{Yb}(\mathrm{OTf})_{3}(248 \mathrm{mg}, 400 \mu \mathrm{mol}, 10 \mathrm{~mol} \%)$ in THF $(24 \mathrm{~mL})$ were reacted according to the GP. Silica gel column chromatography ( $n$-pentane/EtOAc 2:1 $\rightarrow 1: 2$ ) gave the desired $(S, S)$-diastereomer $7 \mathrm{~g}(350 \mathrm{mg}, 1.17 \mathrm{mmol}, 32 \%)$ as yellow oil. ${ }^{1} \mathrm{H}$ NMR $\left(300 \mathrm{MHz}, \mathrm{CDCl}_{3}\right) \delta 1.83-1.98(\mathrm{~m}, 1 \mathrm{H}), 2.07(\mathrm{~s}, 3 \mathrm{H})$, 2.19 (br s, 1H, NH), 2.24-2.38 (m, 1H), 2.58-2.72 (m, 2H), $2.75(\mathrm{~s}, 3 \mathrm{H}), 3.75-3.89(\mathrm{~m}, 1 \mathrm{H}), 6.03(\mathrm{br} \mathrm{s}, 1 \mathrm{H}), 7.45-7.60(\mathrm{~m}$, $4 \mathrm{H}), 7.85-7.97(\mathrm{~m}, 3 \mathrm{H}), 8.20-8.25(\mathrm{~m}, 1 \mathrm{H}) ;{ }^{13} \mathrm{C} \mathrm{NMR}$ $\left(75 \mathrm{MHz}, \mathrm{CDCl}_{3}\right) \delta 15.2,28.0,30.6,31.5,58.7,77.2,122.6$, $125.4,126.2,126.9,129.0,129.9,131.2,133.6,134.1,175.5$ (one aromatic CH-signal is missing); $\alpha_{\mathrm{D}}^{24.0}\left(c 1.0, \mathrm{CHCl}_{3}\right)$ 
$+66.0^{\circ}$; IR (ATR) $\tilde{v}\left(\mathrm{~cm}^{-1}\right): 3321,2957,1689,1396,1322$; HRMS (ESI) $m / z$ : calcd for $\mathrm{C}_{17} \mathrm{H}_{20} \mathrm{~N}_{2} \mathrm{OS}, 323.1189$; found, 323.1191 .

(2R,5S)-5-Benzyl-3-methyl-2-(pyren-1-yl)imidazolidin-4-one $((R, S)-3 b)$ and $(2 S, 5 S)-5$-benzyl-3-methyl-2-(pyren-1yl)imidazolidin-4-one $((\boldsymbol{S}, \boldsymbol{S})-\mathbf{3 b})$. $(S)$-Phenylalanine methyl amide $(0.89 \mathrm{~g}, 5.0 \mathrm{mmol}, 1.0$ equiv), pyrene-1-carbaldehyde (1.73g, $7.50 \mathrm{mmol}, 1.5$ equiv), $4 \AA$ molecular sieves $(400 \mathrm{mg})$ and $\mathrm{Yb}(\mathrm{OTf})_{3}(310 \mathrm{mg}, 500 \mu \mathrm{mol}, 10 \mathrm{~mol} \%)$ in THF $(20 \mathrm{~mL})$ were reacted according to the GP. Silica gel column chromatography ( $n$-pentane/EtOAc $4: 1 \rightarrow 2: 1 \rightarrow 1: 2$ ) gave the faster eluting $(R, S)$-diastereomer $(R, S)$-3b (801 mg, $2.05 \mathrm{mmol}, 41 \%)$ and the desired $(S, S)$-diastereomer $(S, S)-\mathbf{3 b}(952 \mathrm{mg}$, $2.44 \mathrm{mmol}, 49 \%)$ as yellow solids. Analytical data of $(R, S)-\mathbf{3 b}$ : mp $70{ }^{\circ} \mathrm{C} ;{ }^{1} \mathrm{H}$ NMR $\left(600 \mathrm{MHz}, \mathrm{CDCl}_{3}\right) \delta 2.36($ br s, $1 \mathrm{H}), 2.70$ (s, 3H), 3.10-3.24 (m, 2H), $4.15(\mathrm{dd}, J=5.2,5.2 \mathrm{~Hz}, 1 \mathrm{H}), 5.74$ (s, 1H), 7.29 (ddt, $J=9.2,7.4,1.2 \mathrm{~Hz}, 1 \mathrm{H}), 7.34-7.39(\mathrm{~m}, 2 \mathrm{H})$, $7.41-7.45$ (m, 2H), $7.82(\mathrm{~d}, J=7.4 \mathrm{~Hz}, 1 \mathrm{H}), 8.02-8.07$ (m, 2H), $8.10(\mathrm{~d}, J=9.2 \mathrm{~Hz}, 1 \mathrm{H}), 8.15-8.24(\mathrm{~m}, 5 \mathrm{H}) ;{ }^{13} \mathrm{C}$ NMR $(150$ $\left.\mathrm{MHz}, \mathrm{CDCl}_{3}\right) \delta 27.9,38.8,60.2,121.6,122.8,124.6,125.1$, $125.2,125.5,125.8,126.3,126.8,127.2,128.1,128.4,128.6$, $129.1,129.9,130.5,131.2,131.2,131.7,137.7,175.4$ (one aliphatic $\mathrm{CH}$-signal is missing); $\alpha_{\mathrm{D}}^{24.0}\left(c 1.0, \mathrm{CHCl}_{3}\right)-62.0^{\circ}$; IR (ATR) $\tilde{v}\left(\mathrm{~cm}^{-1}\right): 3310,3029,1688,1395,1086$; HRMS (ESI) $m / z$ : calcd for $\mathrm{C}_{27} \mathrm{H}_{22} \mathrm{~N}_{2} \mathrm{O}, 413.1624$; found, 413.1626. Analytical data of (S,S)-3b: mp $95{ }^{\circ} \mathrm{C} ;{ }^{1} \mathrm{H}$ NMR $\left(600 \mathrm{MHz}, \mathrm{CDCl}_{3}\right) \delta$ 2.68 (s, 3H), 3.18 (br d, $J=14.2 \mathrm{~Hz}, 1 \mathrm{H}), 3.36$ (dd, $J=14.2,5.4$ $\mathrm{Hz}, 1 \mathrm{H}), 4.04$ (t, $J=5.4 \mathrm{~Hz}, 1 \mathrm{H}), 6.32$ (br s, 1H), 7.17-7.25 (m, 2H), 7.24-7.31 (m, 5H), 7.95-8.01 (m, 1H), 8.01-8.08 (m, 2H), $8.11(\mathrm{~d}, J=8.9 \mathrm{~Hz}, 1 \mathrm{H}), 8.19-8.25(\mathrm{~m}, 2 \mathrm{H}), 8.28-8.39(\mathrm{~m}, 1 \mathrm{H})$; ${ }^{13} \mathrm{C}$ NMR $\left(75 \mathrm{MHz}, \mathrm{CDCl}_{3}\right) \delta 27.9,36.7,60.9,121.3,124.6$, $124.8,125.3,125.5,125.8,126.2,127.0,127.2$, 128.2, 128.6, $128.9,129.4,129.8,130.5,131.2,136.5,175.0$ (one aliphatic, two aromatic $\mathrm{CH}$-signals as well as one aromatic $\mathrm{C}_{\mathrm{q}}$-signal are missing); $\alpha_{\mathrm{D}}^{24.0}\left(\right.$ c 1.0, $\left.\mathrm{CHCl}_{3}\right)-10.0^{\circ}$; IR (ATR) $\tilde{v}\left(\mathrm{~cm}^{-1}\right)$ : 3475, 2914, 1682, 1255, 1033; HRMS (ESI) $\mathrm{m} / \mathrm{z}$ : calcd for $\mathrm{C}_{27} \mathrm{H}_{22} \mathrm{~N}_{2} \mathrm{O}$, 413.1624; found, 413.1627 .

(2S,5S)-5-Benzyl-3-methyl-2-mesitylimidazolidin-4-one (3c). $(S)$-Phenylalanine methyl amide $(71.3 \mathrm{mg}, 400 \mu \mathrm{mol}$, 1.0 equiv), mesityl carbaldehyde $(65.2 \mathrm{mg}, 64.9 \mu \mathrm{L}, 440 \mu \mathrm{mol}$, 1.1 equiv), $4 \AA$ molecular sieves (40 mg) and $\mathrm{Yb}(\mathrm{OTf})_{3}(25 \mathrm{mg}$, $40 \mu \mathrm{mol}, 10 \mathrm{~mol} \%)$ in THF $(2.0 \mathrm{~mL})$ were reacted according to the GP. Silica gel column chromatography ( $n$-pentane/EtOAc $3: 1 \rightarrow 1: 1)$ gave the desired $(S, S)$-diastereomer 3c $(64.1 \mathrm{mg}$, $208 \mu \mathrm{mol}, 52 \%)$ as a white solid: $\mathrm{mp} 75{ }^{\circ} \mathrm{C}$; ${ }^{1} \mathrm{H}$ NMR $\left(600 \mathrm{MHz}, \mathrm{CDCl}_{3}\right) \delta 1.80(\mathrm{~s}, 3 \mathrm{H}), 2.24(\mathrm{~s}, 3 \mathrm{H}), 2.37(\mathrm{~s}, 3 \mathrm{H})$, $2.58(\mathrm{~s}, 3 \mathrm{H}), 3.18(\mathrm{ddd}, J=14.0,6.9,0.9 \mathrm{~Hz}, 1 \mathrm{H}), 3.24$ (ddd, $J$ $=14.0,3.8,0.9 \mathrm{~Hz}, 1 \mathrm{H}), 3.83(\mathrm{ddd}, J=6.9,3.8,1.7 \mathrm{~Hz}, 1 \mathrm{H})$, $5.72(\mathrm{~d}, J=1.7 \mathrm{~Hz}, 1 \mathrm{H}), 6.75(\mathrm{~s}, 1 \mathrm{H}), 6.83(\mathrm{~s}, 1 \mathrm{H}), 7.20-7.24$ $(\mathrm{m}, 1 \mathrm{H}), 7.25-7.28(\mathrm{~m}, 4 \mathrm{H}) ;{ }^{13} \mathrm{C} \mathrm{NMR}\left(150 \mathrm{MHz}, \mathrm{CDCl}_{3}\right) \delta$ $18.4,20.3,20.6,26.8,35.8,60.6,72.8,126.7,128.2,128.5$, $129.5,129.6,131.5,137.1,137.4,137.9,138.4,173.8 ; \alpha_{\mathrm{D}}^{24.0}$ $\left(c 1.0, \mathrm{CHCl}_{3}\right)-62.0^{\circ}$; IR (ATR) $\tilde{v}\left(\mathrm{~cm}^{-1}\right): 3315,2921,1693$, 1433, 1309; HRMS (ESI) $m / z$ : calcd for $\mathrm{C}_{20} \mathrm{H}_{24} \mathrm{~N}_{2} \mathrm{O}$, 331.1781; found, 331.1781 .

(2S,5S)-5-Benzyl-3-methyl-2-(2,4,6-triisopropylphen-1yl)imidazolidin-4-one (3d). ( $S$ )-Phenylalanine methyl amide (217 mg, $1.00 \mu \mathrm{mol}, 1.0$ equiv), 2,4,6-triisopropylbenzaldehyde (255 mg, $273 \mu \mathrm{L}, 1.10 \mathrm{mmol}, 1.1$ equiv), $4 \AA$ molecular sieves $(80 \mathrm{mg})$ and $\mathrm{Yb}(\mathrm{OTf})_{3}(62.1 \mathrm{mg}, 100 \mu \mathrm{mol}, 10 \mathrm{~mol} \%)$ in THF $(4.0 \mathrm{~mL})$ were reacted according to the GP. Silica gel column chromatography ( $n$-pentane/EtOAc 10:1 $\rightarrow 2: 1)$ gave the desired $(S, S)$-diastereomer 3d $(90.2 \mathrm{mg}, 230 \mu \mathrm{mol}, 23 \%)$ as a colorless oil: ${ }^{1} \mathrm{H}$ NMR $\left(600 \mathrm{MHz}, \mathrm{CDCl}_{3}\right) \delta 1.13(\mathrm{~d}, J=$ $6.9 \mathrm{~Hz}, 3 \mathrm{H}), 1.15(\mathrm{~d}, J=6.9 \mathrm{~Hz}, 3 \mathrm{H}), 1.24(\mathrm{~d}, J=6.9 \mathrm{~Hz}, 12 \mathrm{H})$, $2.69(\mathrm{~s}, 3 \mathrm{H}), 2.83-2.90(\mathrm{~m}, 2 \mathrm{H}), 3.30$ (hept, $J=6.9 \mathrm{~Hz}, 1 \mathrm{H})$, 3.41 (dd, $J=14.1,3.4 \mathrm{~Hz}, 1 \mathrm{H}), 3.46$ (hept, $J=6.9 \mathrm{~Hz}, 1 \mathrm{H}$ ), $3.84(\mathrm{~d}, J=8.7 \mathrm{~Hz}, 1 \mathrm{H}), 5.92($ br s, $1 \mathrm{H}), 7.00$ (d, $J=1.9 \mathrm{~Hz}$, $1 \mathrm{H}), 7.07(\mathrm{~d}, J=1.9 \mathrm{~Hz}, 1 \mathrm{H}), 7.17-7.25(\mathrm{~m}, 1 \mathrm{H}), 7.26-7.30(\mathrm{~m}$, $4 \mathrm{H}) ;{ }^{13} \mathrm{C} \mathrm{NMR}\left(150 \mathrm{MHz}, \mathrm{CDCl}_{3}\right) \delta 23.8,23.8,24.2,24.3$, 24.7, 25.7, 27.5, 27.5, 29.1, 34.1, 37.7, 61.3, 71.5, 121.2, 123.6, 126.0, 126.6, 128.6, 129.2, 138.2, 148.4, 149.7, 150.1, 173.7; $\alpha_{\mathrm{D}}^{24.0}\left(c\right.$ 1.0, $\left.\mathrm{CHCl}_{3}\right)-102^{\circ}$; IR (ATR) $\tilde{v}\left(\mathrm{~cm}^{-1}\right): 3548,2957$, 1683, 1397, 1098; HRMS (ESI) $m / z$ : calcd for $\mathrm{C}_{26} \mathrm{H}_{36} \mathrm{~N}_{2} \mathrm{O}$, 415.2720; found, 415.2722.

\section{Supporting Information}

\section{Supporting Information File 1}

Copies of ${ }^{1} \mathrm{H}$ and ${ }^{13} \mathrm{C}$ NMR spectra.

[http://www.beilstein-journals.org/bjoc/content/ supplementary/1860-5397-13-254-S1.pdf]

\section{References}

1. Knoevenagel, E. Ber. Dtsch. Chem. Ges. 1898, 31, 2596-2619. doi:10.1002/cber.18980310308

2. Erkkilä, A.; Majander, I.; Pihko, P. M. Chem. Rev. 2007, 107, 5416-5470. doi:10.1021/cr068388p

3. Mukherjee, S.; Yang, J. W.; Hoffmann, B.; List, B. Chem. Rev. 2007, 107, 5471-5569. doi:10.1021/cr0684016

4. Moyano, A.; Rios, R. Chem. Rev. 2011, 111, 4703-4832. doi:10.1021/cr100348t

5. Nielsen, M.; Worgull, D.; Zweifel, T.; Gschwend, B.; Bertelsen, S.; Jørgensen, K. A. Chem. Commun. 2011, 47, 632-649. doi:10.1039/COCC02417A

6. Torres, R. R., Ed. Stereoselective Organocatalysis: Bond Formation Methodologies and Activation Modes; Wiley-VCH: Weinheim, Germany, 2013. doi:10.1002/9781118604755

7. Hayashi, Y. Chem. Sci. 2016, 7, 866-880. doi:10.1039/C5SC02913A 
8. Woodward, R. B.; Logusch, E.; Nambiar, K. P.; Sakan, K.; Ward, D. E.; Au-Yeung, B.-W.; Balaram, P.; Browne, L. J.; Card, P. J.; Chen, C. H.; Chênevert, R. B.; Fliri, A.; Frobel, K.; Gais, H.-J.; Garratt, D. G.; Hayakawa, K.; Heggie, W.; Hesson, D. P.; Hoppe, D.; Hoppe, I.; Hyatt, J. A.; Ikeda, D.; Jacobi, P. A.; Kim, K. S.; Kobuke, Y.; Kojima, K.; Krowicki, K.; Lee, V. J.; Leutert, T.; Malchenko, S.; Martens, J.; Matthews, R. S.; Ong, B. S.; Press, J. B.; Rajan Babu, T. V.; Rousseau, G.; Sauter, H. M.; Suzuki, M.; Tatsuta, K.; Tolbert, L. M.; Truesdale, E. A.; Uchida, I.; Ueda, Y.; Uyehara, T.; Vasella, A. T.; Vladuchick, W. C.; Wade, P. A.; Williams, R. M.; Wong, H. N.-C. J. Am. Chem. Soc. 1981, 103, 3210-3213. doi:10.1021/ja00401a049

9. Yamaguchi, M.; Yokota, N.; Minami, T. J. Chem. Soc., Chem. Commun. 1991, 1088-1089. doi:10.1039/C39910001088

10. Zhuang, W.; Marigo, M.; Jørgensen, K. A. Org. Biomol. Chem. 2005, 3, 3883-3885. doi:10.1039/b512542a

11. Marigo, M.; Wabnitz, T. C.; Fielenbach, D.; Jørgensen, K. A. Angew. Chem., Int. Ed. 2005, 44, 794-797. doi:10.1002/anie.200462101

12. Hayashi, Y.; Gotoh, H.; Hayashi, T.; Shoji, M. Angew. Chem., Int. Ed. 2005, 44, 4212-4215. doi:10.1002/anie.200500599

13. Ahrendt, K. A.; Borths, C. J.; MacMillan, D. W. C. J. Am. Chem. Soc. 2000, 122, 4243-4244. doi:10.1021/ja000092s

14. Jen, W. S.; Wiener, J. J. M.; MacMillan, D. W. C. J. Am. Chem. Soc. 2000, 122, 9874-9875. doi:10.1021/ja005517p

15. Paras, N. A.; MacMillan, D. W. C. J. Am. Chem. Soc. 2001, 123, 4370-4371. doi:10.1021/ja015717g

16. Brown, S. P.; Goodwin, N. C.; MacMillan, D. W. C. J. Am. Chem. Soc. 2003, 125, 1192-1194. doi:10.1021/ja029095q

17. Harmata, M.; Ghosh, S. K.; Hong, X.; Wacharasindhu, S.; Kirchhoefer, P. J. Am. Chem. Soc. 2003, 125, 2058-2059. doi:10.1021/ja029058z

18. Mangion, I. K.; Northrup, A. B.; MacMillan, D. W. C. Angew. Chem., Int. Ed. 2004, 43, 6722-6724. doi:10.1002/anie.200461851

19. Fjelbye, K.; Marigo, M.; Clausen, R. P.; Juhl, K. Org. Lett. 2016, 18, 1170-1173. doi:10.1021/acs.orglett.6b00293

20. Yang, M.; Yang, X.; Sun, H.; Li, A. Angew. Chem., Int. Ed. 2016, 55, 2851-2855. doi:10.1002/anie.201510568

21.Sun, W.-B.; Wang, X.; Sun, B.-F.; Zou, J.-P.; Lin, G.-Q. Org. Lett. 2016, 18, 1219-1221. doi:10.1021/acs.orglett.6b00150

22. Xie, Z.; Zan, X.; Sun, S.; Pan, X.; Liu, L. Org. Lett. 2016, 18, 3944-3947. doi:10.1021/acs.orglett.6b01625

23. Holland, M. C.; Metternich, J. B.; Daniliuc, C.; Schweizer, W. B.; Gilmour, R. Chem. - Eur. J. 2015, 21, 10031-10038. doi:10.1002/chem.201500270

24. Welin, E. R.; Warkentin, A. A.; Conrad, J. C.; MacMillan, D. W. C. Angew. Chem., Int. Ed. 2015, 54, 9668-9672. doi:10.1002/anie.201503789

25. Wallbaum, J.; Garve, L. K. B.; Jones, P. G.; Werz, D. B. Chem. - Eur. J. 2016, 22, 18756-18759. doi:10.1002/chem.201605265

26. Paras, N. A.; MacMillan, D. W. C. J. Am. Chem. Soc. 2002, 124 , 7894-7895. doi:10.1021/ja025981p See as an example of an experimental procedure.

27. Jain, R.; Cohen, L. A. Tetrahedron 1996, 52, 5363-5370. doi:10.1016/0040-4020(96)00187-1

28. Daka, P.; Liu, A.; Karunaratne, C.; Csatary, E.; Williams, C.; Xiao, H.; Lin, J.; Xu, Z.; Page, R. C.; Wang, H. Bioorg. Med. Chem. 2015, 23 , 1348-1355. doi:10.1016/j.bmc.2015.01.025
29. Magnus, P.; Mugrage, B.; DeLuca, M. R.; Cain, G. A.

J. Am. Chem. Soc. 1990, 112, 5220-5230. doi:10.1021/ja00169a033

30. Samulis, L.; Tomkinson, N. C. O. Tetrahedron 2011, 67, 4263-4267. doi:10.1016/j.tet.2011.04.009

\section{License and Terms}

This is an Open Access article under the terms of the Creative Commons Attribution License (http://creativecommons.org/licenses/by/4.0), which permits unrestricted use, distribution, and reproduction in any medium, provided the original work is properly cited.

The license is subject to the Beilstein Journal of Organic Chemistry terms and conditions:

(http://www.beilstein-journals.org/bjoc)

The definitive version of this article is the electronic one which can be found at: doi:10.3762/bjoc. 13.254 